\title{
Effects of Natural Eggshell Membrane (NEM) on Cytokine Production in Cultures of Peripheral Blood Mononuclear Cells: Increased Suppression of Tumor Necrosis Factor- $\alpha$ Levels After In Vitro Digestion
}

\author{
Kathleen F. Benson, ${ }^{1}$ Kevin J. Ruff, ${ }^{2}$ and Gitte S. Jensen ${ }^{1}$ \\ ${ }^{1}$ NIS Labs, Klamath Falls, Oregon, USA. \\ ${ }^{2}$ ESM Technologies, LLC, Carthage, Missouri, USA.
}

\begin{abstract}
Tumor necrosis factor $-\alpha(\mathrm{TNF}-\alpha)$ plays an important role in inflammatory processes. This study examined the effects of natural eggshell membrane (NEM ${ }^{\circledR}$ ) (ESM Technologies, LLC, Carthage, MO, USA) on interleukin (IL)-2, IL-4, IL6 , IL-10, interferon- $\gamma$ (IFN- $\gamma$ ), and TNF- $\alpha$ cytokine production by 4-day peripheral blood mononuclear cell (PBMC) cultures exposed to serial dilutions of either an aqueous extract of natural eggshell membrane (NEM-AQ) or NEM subjected to in vitro digestion (NEM-IVD). The effects on cytokine production were also assessed in the presence of phytohemagglutinin (PHA) and pokeweed mitogen (PWM) where exposure to NEM-AQ resulted in reduced levels of proliferation and statistically significant effects on IL-6, IL-10, IFN- $\gamma$, and TNF- $\alpha$ cytokine production. NEM-AQ reduced levels of IL-6, IL-10, IFN- $\gamma$, and TNF- $\alpha$ in cultures exposed to PHA. In cultures containing PWM, NEM-AQ reduced production of IL-10 and at the highest dose tested increased IL-6 and decreased TNF- $\alpha$ cytokine levels. NEM-IVD, at the two lowest concentrations of product, significantly reduced TNF- $\alpha$ production by PBMC cultures exposed to PWM compared with the in vitro digest control or native NEM. Taken together, these results suggest that NEM-AQ can influence signaling events in response to the T cellspecific mitogen PHA as well as to the mitogen PWM that require cellular cross-talk and that these effects may be partially mediated through a reduction in level of the pro-inflammatory cytokine TNF- $\alpha$. The suppression of TNF- $\alpha$ production in the presence of NEM-IVD is promising for the use of NEM as a consumable anti-inflammatory product.
\end{abstract}

KEY WORDS: • cytokines $\bullet$ human $\bullet$ immunity $\bullet$ in vitro digestion $\bullet$ lymphocyte $\bullet$ natural eggshell membrane $\bullet$ natural product $\bullet$ peripheral blood mononuclear cells $\bullet$ Th1/Th2 $\bullet$ tumor necrosis factor- $\alpha$

\section{INTRODUCTION}

$\mathbf{T}$ HE MAIN CLINICAL MANIFESTATIONS of arthritis are inflammation, pain, and bone resorption. Chronic inflammation and bone loss are closely linked pathophysiologic events. New scientific data point to a beneficial effect of blocking specific molecular interactions, which can reduce local arthritic symptoms even in the presence of ongoing chronic inflammation. ${ }^{1}$ The current mainstream medical treatments for arthritis involve pain management, anti-inflammatory drugs (nonsteroidal anti-inflammatory drugs, steroids, cyclooxygenase-2 inhibitors), and also exploration of chemokine receptor antagonists to stop cell migration into the inflamed areas. ${ }^{2-4}$ Part of the intensive pharmaceutical research efforts includes research on the interaction between osteoblasts and osteoclasts via the receptor activator of nuclear factor $\kappa \mathrm{B}$ and its ligand. Receptor activator of nuclear factor $\kappa$ is a hematopoietic surface receptor controlling osteoclastogenesis and calcium metabo-

Manuscript received 26 December 2010. Revision accepted 10 October 2011.

Address correspondence to: Gitte S. Jensen, NIS Labs, 1437 Esplanade, Klamath Falls, OR 97601, USA, E-mail: gitte@nislabs.com lism. Interference with these various pathways may also include arresting the maturation of phagocytic mononuclear cells into bone-resorbing cells, neutralizing pro-inflammatory cytokines, and blocking of matrix metalloproteinases. These mainstream treatments go far beyond a direct treatment of cells within the arthritic joints. They aim at reducing inflammation and inhibiting recruitment into the inflamed area of cells that contribute to disease processes, including bone resorption.

In contrast, nutraceutical products widely used for joint health include glucosamine, chondroitin, and hyaluronic acid, thus ignoring a multifaceted action of complex natural products. Even the spotlight on hyaluronic acid seems to limit its focus on replenishing the synovial fluid and on stimulating chondrocytes to produce more hyaluronic acid, thus ignoring the many complex ways that hyaluronic acid can modulate cells and their behavior.

Natural eggshell membrane $\left(\mathrm{NEM}^{\circledR}\right)$ (ESM Technologies, LLC, Carthage, MO, USA) is a novel dietary supplement that has been shown in several human trials to be a clinically effective treatment for pain and stiffness associated with joint and connective tissue disorders, particularly osteoarthritis. ${ }^{5,6}$ Eggshell membrane is primarily composed 
of fibrous proteins such as collagen type $\mathrm{I}^{7}$ However, eggshell membranes have also been shown to contain glycosaminoglycans, such as dermatan sulfate, chondroitin sulfate, ${ }^{8}$ and hyaluronic acid, ${ }^{9}$ and sulfated glycoproteins, including hexosamines such as glucosamine. ${ }^{10}$ NEM contains up to about $5 \%$ of these various components, the unique combination of which may explain its biological activity. To further this understanding, a cytokine profile was determined from NEM-treated lymphocytes in cell culture.

The purpose of this study is to evaluate anti-inflammatory and immunomodulatory effects of NEM as well as its in vitro digest in a select series of human cell-based in vitro assays, in preparation for more comprehensive evaluations in vitro and in vivo.

\section{MATERIALS AND METHODS}

\section{Reagents}

Phosphate-buffered saline (PBS) (pH 7.4), RPMI-1640 culture medium, fetal calf serum, L-glutamine $(200 \mathrm{mM})$, penicillin-streptomycin $(100 \times$ solution $)$, glacial acetic acid, methanol, pepsin, pancreatin, bile salts, Histopaque 1077, and Histopaque 1119 were obtained from Sigma-Aldrich (St. Louis, MO, USA). The cytometric bead array (CBA) for human Th1/Th2 cytokine kit II was purchased from BD Biosciences (San Jose, CA, USA). All reagents for sodium dodecyl sulfate (SDS)-polyacrylamide gel electrophoresis (PAGE) and silver stain detection were obtained from BioRad (Hercules, CA, USA) and included 4-15\% Tris- $\mathrm{HCl}$ ready gels, Precision Plus dual color protein molecular weight standards, Laemmli buffer, $10 \times$ Tris/glycine/SDS buffer, silver stain concentrate, silver stain oxidizer, and silver stain developer. NEM was obtained from ESM Technologies, LLC.

\section{Preparation of NEM for in vitro bioassays}

The NEM powder was reconstituted in physiological saline and allowed to rehydrate for 1 hour at room temperature. Solids included insoluble calcium carbonate from eggshell and were removed by centrifugation at $900 \mathrm{~g}$ for 10 minutes. The liquid was filtered through a sterile cellulose acetate syringe filter (pore size, $0.22 \mu \mathrm{m}$ ). This filtrate corresponded to a stock solution of $100 \mathrm{~g} / \mathrm{L}$ product (same concentration as the in vitro-digested NEM [NEM-IVD], described below). This aqueous preparation is designated NEM-AQ.

\section{Preparation of an in vitro digest of NEM}

The in vitro digestion of NEM was performed according to methods published in the literature ${ }^{11-13}$ (see also Fig. 4). In brief, $3.75 \mathrm{~g}$ of NEM powder was added to $30 \mathrm{~mL}$ of PBS and shaken at room temperature for 1 hour. Following the 1hour incubation, the sample was spun at $900 \mathrm{~g}$ for $10 \mathrm{~min}$ utes, and the aqueous solution was removed from the solids and sterile-filtered with a cellulose acetate filter (pore size, $0.22 \mu \mathrm{m}) . \mathrm{HCl}(1 M)$ was then added until the solution reached $\mathrm{pH}$ 2.0. Porcine pepsin was then added at a con- centration of $1.3 \mathrm{mg} / \mathrm{mL}$, and the sample was left at $37^{\circ} \mathrm{C}$ for 60 minutes with shaking (to simulate digestion in the stomach). Next, sodium bicarbonate $\left(\mathrm{NaHCO}_{3}\right)$ was used to increase the $\mathrm{pH}$ of the solution to 5.8 (irreversibly inactivating pepsin), and pancreatin $(0.175 \mathrm{mg} / \mathrm{mL})$ and porcine bile salts $(1.1 \mathrm{mg} / \mathrm{mL})$ were added to simulate intestinal digestion. The $\mathrm{pH}$ was then adjusted to 6.5 , and the mixture was left at $37^{\circ} \mathrm{C}$ for 1 hour. The final volume of the digest was adjusted with saline to give a final concentration of NEM of $100 \mathrm{~g} / \mathrm{L}$. Following this in vitro digestion, the sample was centrifuged through a $10-\mathrm{kDa}$ cutoff filtration spin column to remove the enzymes from the digested product. This filtration step was necessary to avoid the presence of digestive enzymes in the downstream treatment of cells with product. This step also avoided the use of enzyme inhibitors that potentially could have direct effects on cell signaling in downstream cell-based assays. The liquid after in vitro digestion and size-exclusion filtration is designated NEM-IVD.

As the control, saline alone (negative control) was simultaneously subjected to the in vitro digestion protocol as described above. This was an important control to determine whether any bile salts or breakdown products from the enzymes themselves have biological activity. This PBS control is designated as PBS-IVD.

\section{SDS-PAGE}

SDS-PAGE was performed to compare crude NEM-AQ, NEM-IVD, and the PBS-IVD control. Samples were denatured by boiling for 3 minutes in $1 \times$ Laemmli buffer and separated by gel electrophoresis through a $4-15 \%$ polyacrylamide Tris- $\mathrm{HCl}$ gel using Protein Plus dual color molecular weight standards for reference. Silver staining was performed in order to visualize proteins, and an image of the stained gel was captured with a Canon (Lake Success, NY, USA) PowerShot SD430 digital camera.

\section{Purification of peripheral blood mononuclear cells}

Healthy human volunteers between the ages of 20 and 50 years served as blood donors after written informed consent was obtained, as approved by the Sky Lakes Medical (Klamath Falls, OR, USA) Center Institutional Review Board. Isolation of peripheral blood mononuclear cells (PBMCs) was performed as previously described. ${ }^{14}$ PBMCs were used to establish lymphocyte cultures for the measurement of cytokine production.

\section{Cytokine production by 4-day PBMC cultures}

Freshly purified PBMCs were resuspended in RPMI 1640 medium supplemented with $10 \%$ fetal bovine serum, Lglutamine $(2 \mathrm{mM})$, penicillin $(100 \mathrm{U} / \mathrm{mL})$, and streptomycin $(100 \mathrm{mg} / \mathrm{mL})$ at a density of $1 \times 10^{6} / \mathrm{mL}$. Cells were cultured in the presence of 10-fold serial dilutions of NEM-AQ, NEM-IVD, or PBS-IVD in a series of triplicate wells containing a total volume of $200 \mu \mathrm{L}$. Three separate sets of 
culture conditions were established: no mitogen, phytohemagglutinin (PHA), or pokeweed mitogen (PWM). The culture plate was incubated at $37^{\circ} \mathrm{C}$ in an atmosphere of $5 \%$ $\mathrm{CO}_{2}$ for 4 days, after which cells were transferred to a V-bottom plate and centrifuged. Cell supernatants were collected for cytokine measurement (described below). Determination of relative cell numbers in each culture well was performed by staining cells with the DNA dye $\mathrm{Cy}$ Quant $^{\circledR}$ (Invitrogen, Carlsbad, CA, USA) and measuring fluorescence measured with a Tecan (Durham, NC, USA) Spectrafluor fluorescence plate reader. Samples were assayed in triplicate, and experiments were repeated three times with cells from three different donors.

\section{Th1/Th2 cytokine profile}

The cytokines interleukin (IL)-2, IL-4, IL-6, IL-10, tumor necrosis factor- $\alpha$ (TNF- $\alpha)$, and interferon- $\gamma$ (IFN- $\gamma)$ were quantified in the 4-day PBMC culture supernatants using a flow cytometry cytometric bead array (CBA) kit (CBA human Th1/Th2 cytokine kit II, BD Biosciences) that allowed the determination of the levels of all six cytokines simultaneously. Samples were tested in duplicate following the manufacturer's instructions, data were acquired with a FACSCalibur $^{\mathrm{TM}}$ flow cytometer (Becton-Dickinson, San Jose), and the analysis was performed with FlowJo software (TreeStar Inc., Ashland, OR, USA).

\section{Statistical analysis}

Statistical significance was tested using Student's $t$ test performed with the program Microsoft (Redmond, WA, USA) Excel. All $P$ values were two-sided and were considered significant when $P<.05$. Only statistically significant $P$ values are reported.

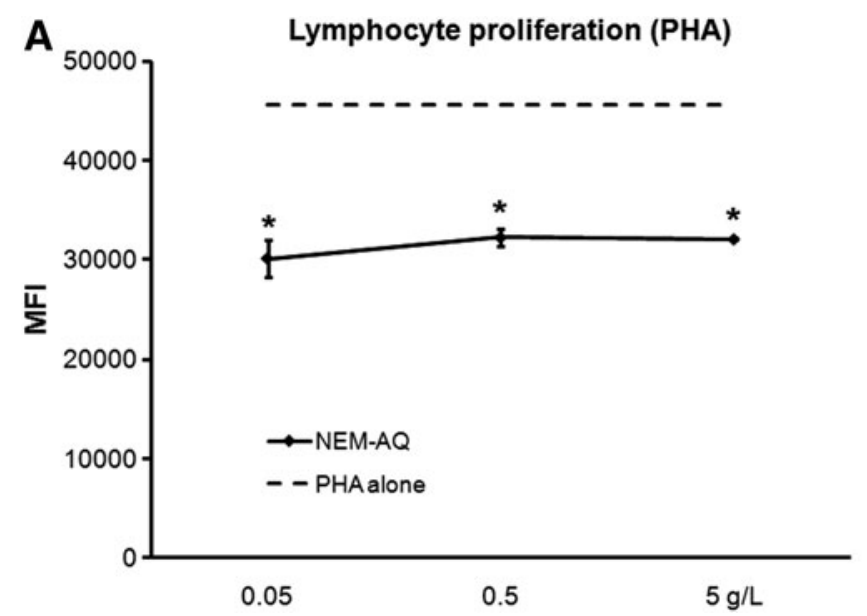

\section{RESULTS}

\section{Lymphocyte proliferation assay}

The lymphocyte proliferation assay evaluates whether a test product alters lymphocyte responsiveness to known signals such as mitogens. If any change in the proliferative response to known mitogens is seen in cells pretreated with test product, this is a good indication that the product has immunomodulatory effects and justifies further in-depth work on $\mathrm{T}$ and $\mathrm{B}$ lymphocyte signaling and activation.

Freshly purified human PBMCs were cultured for 4 days in the absence versus presence of serial dilutions of test products. Three parallel sets of cultures were established, where one tested the direct effect of test product on lymphocyte proliferation, and the two others examined the possible interference with response to the mitogen PHA or PWM. PHA produces a cleaner signal as it strictly induces proliferation of T lymphocytes, but PWM represents a more physiological signal mimicking the cellular interactions between monocytes/macrophages and $\mathrm{T}$ and $\mathrm{B}$ lymphocytes that occur in lymphoid tissue; therefore it is beneficial to test both in parallel. Positive controls included cells treated only with a mitogen in the absence of test product. No direct effects of product on lymphocyte proliferation were observed (data not shown). In the presence of PHA (Fig. 1A) and PWM (Fig. 1B), statistically significant decreases in proliferation of $30 \%$ and $15 \%$, respectively, were seen with all three dilutions of NEM-AQ, indicating that pretreatment of PBMCs with NEM-AQ altered their response to subsequent signals.

\section{Changes in Th1/Th2 cytokine levels}

Supernatants were collected from 4-day cultures where PBMCs were exposed to test products in the absence versus presence of the mitogens PHA and PWM and analyzed for

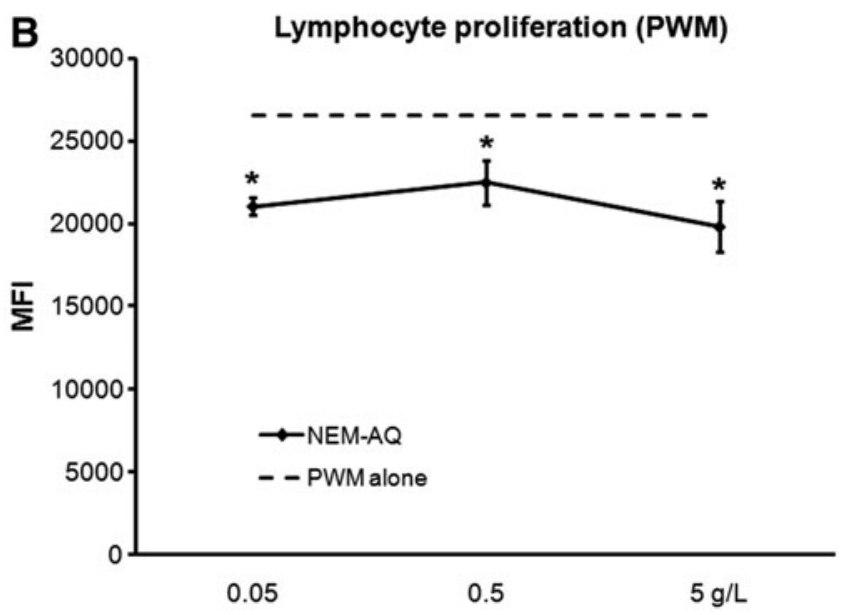

FIG. 1. Effect of aqueous extract of Natural Eggshell Membrane (NEM) (NEM-AQ) on lymphocyte proliferation in the presence of (A) phytohemagglutinin (PHA) and (B) pokeweed mitogen (PWM). Statistically significant decreases in the proliferative response of 4-day lymphocyte cultures to (A) PHA and (B) PWM were seen when cells were exposed to serial dilutions of NEM-AQ. Statistically significant differences are indicated $(* P<.05)$. Conditions were assayed in triplicate, and the results shown are mean \pm SD values from a representative of three separate experiments using cells from three different donors. MFI, mean fluorescence intensity. 
A

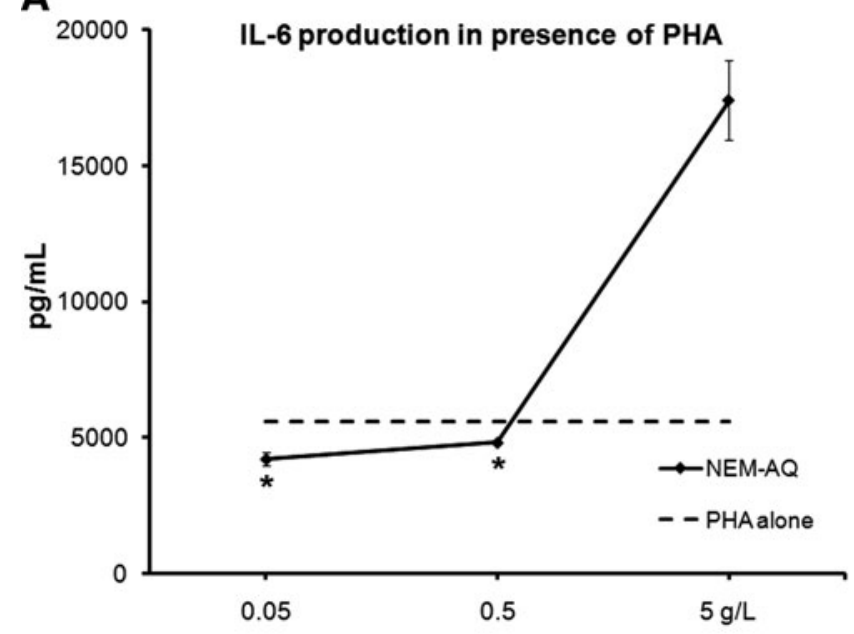

C

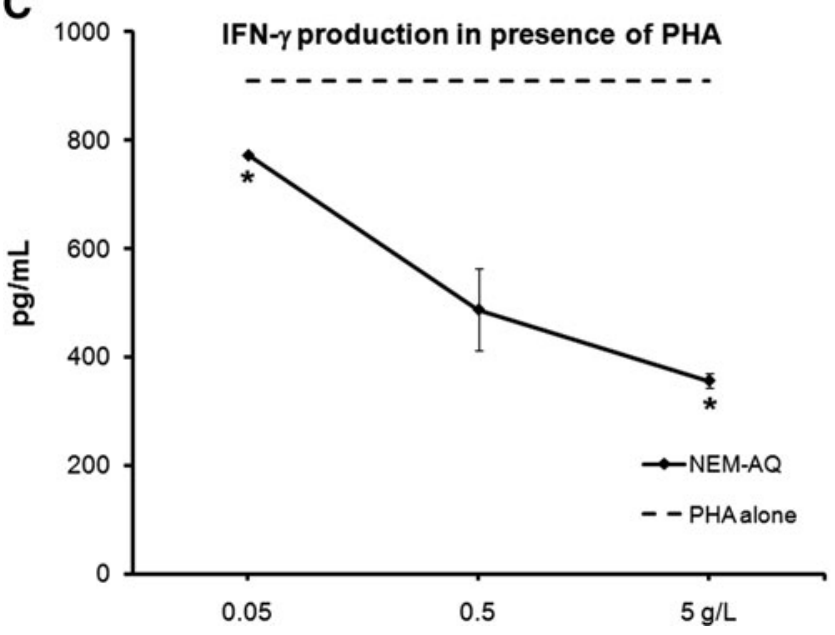

B
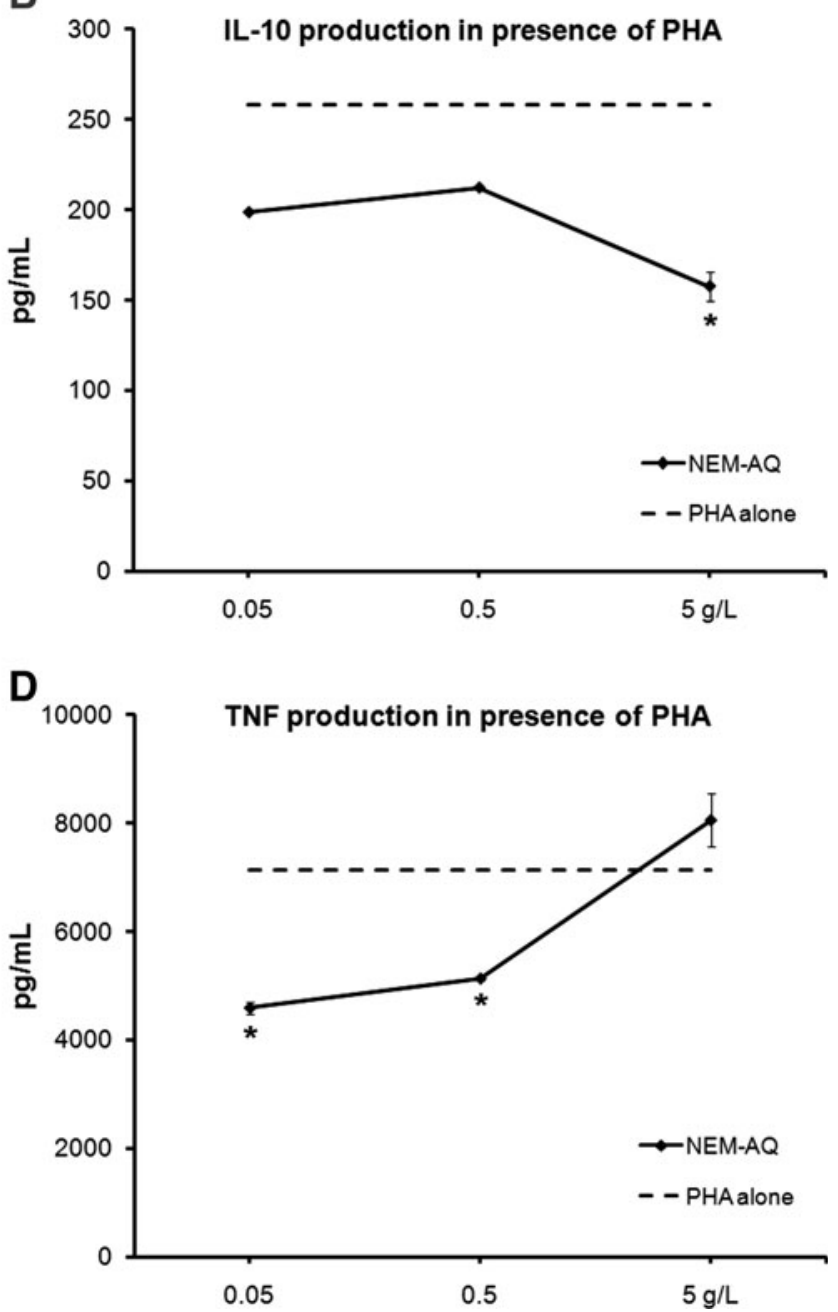

FIG. 2. Effects of NEM-AQ on production of the cytokines (A) interleukin (IL)-6, (B) IL-10, (C) interferon- $\gamma$ (IFN- $\gamma$ ), and (D) tumor necrosis factor- $\alpha(\mathrm{TNF}-\alpha)$ by 4-day peripheral blood mononuclear cell cultures simultaneously exposed to PHA. Supernatants from 4-day peripheral blood mononuclear cell cultures were simultaneously assayed for the presence of the cytokines IL-6, IL-10, IFN- $\gamma$, and TNF- $\alpha$ using a flow cytometrybased assay. (A) IL-6 production in cultures exposed to serial dilutions of NEM-AQ showed a strong increase at the highest dose tested (5 g/L) and a decrease at the two lowest concentrations of NEM-AQ tested. (B) Decreases in IL-10 production were seen with all three doses of NEM-AQ in the presence of PHA. (C) The cytokine IFN- $\gamma$ levels decreased in a dose-dependent manner. This reduction in IFN- $\gamma$ production was over $60 \%$ at the highest concentration of NEM-AQ tested. (D) TNF- $\alpha$ production decreased in cultures exposed to serial dilutions of NEM-AQ. Statistically significant differences are indicated $\left({ }^{*} P<.05\right)$. The results shown are mean \pm SD values from a representative of three separate lymphocyte proliferation cultures using cells from three different donors.

the panel of Th1/Th2 cytokines IL-2, IL-4, Il-6, IL-10, IFN$\gamma$, and TNF- $\alpha$, using a CBA for flow cytometry. No statistically significant changes in cytokine production occurred in unstimulated cultures, whereas statistically significant changes in the cytokines IL-6, IL-10, IFN- $\gamma$, and TNF- $\alpha$ occurred in cultures that contained PHA (Fig. 2) or PWM (Fig. 3).

Cultures exposed to serial dilutions of NEM-AQ showed a biphasic response, including a strong increase of IL-6 at the highest dose tested $(5 \mathrm{~g} / \mathrm{L})$ in the presence of both PHA and PWM, a decrease at the lower concentrations for PHA, and no effect at lower doses for PWM. Levels of IL-10, IFN$\gamma$, and TNF- $\alpha$ decreased in the presence of all three doses of NEM-AQ in the presence of PHA. Effects in the presence of
PWM were only seen at higher doses. The reduction in IFN$\gamma$ production in the presence of PHA was over $60 \%$ at the highest concentration of NEM-AQ tested. At the $0.05 \mathrm{~g} / \mathrm{L}$ dose, a $35 \%$ reduction in TNF- $\alpha$ levels was seen, indicating a strong anti-inflammatory effect of NEM-AQ on TNF- $\alpha$ production at lower concentrations in the presence of the Tcell mitogen PHA.

In vitro digest

An in vitro digest as outlined in Figure 4 was performed on NEM-AQ as well as a saline control (PBS-IVD), and the resulting material was subjected to SDS-PAGE and silver stain detection in parallel with NEM-AQ. This analysis 
A
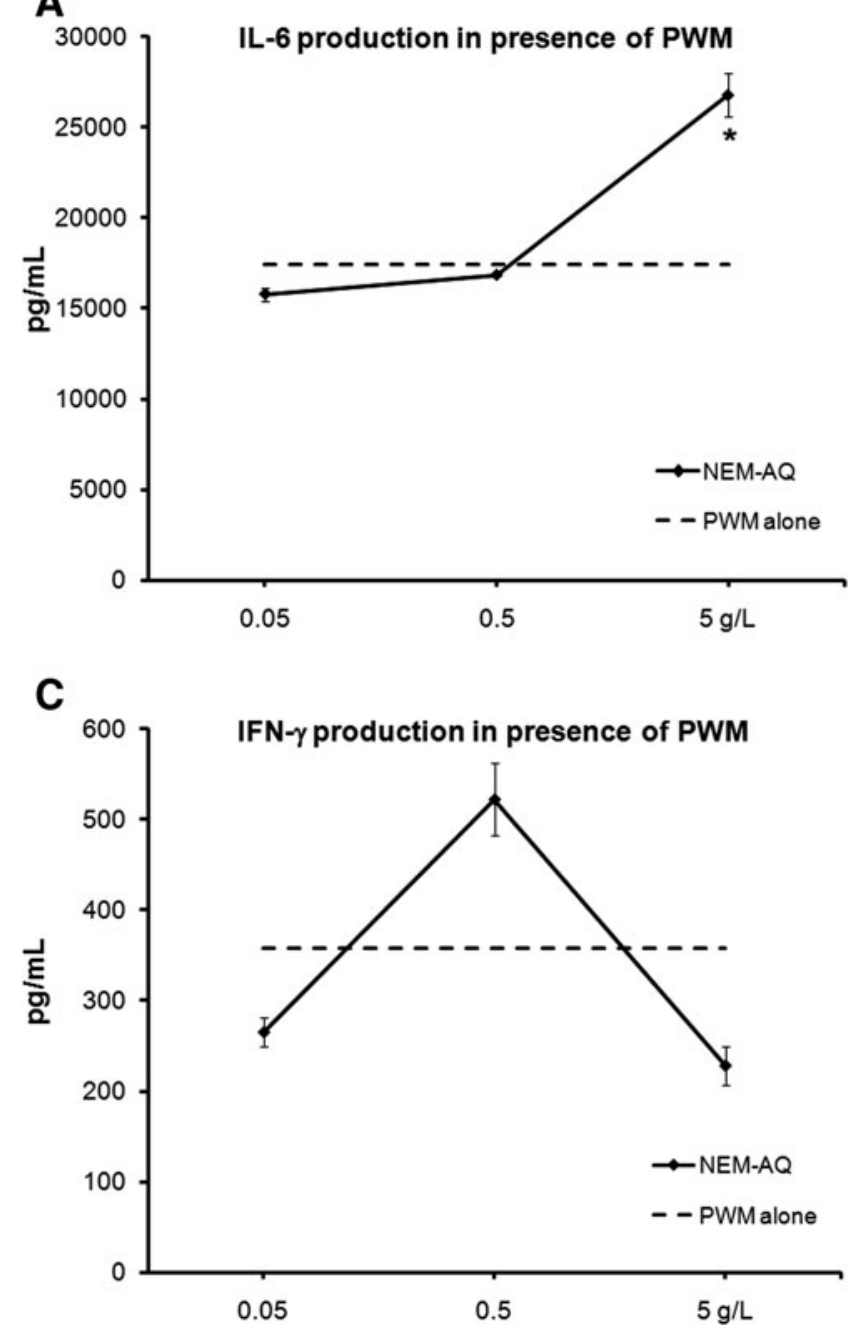

B

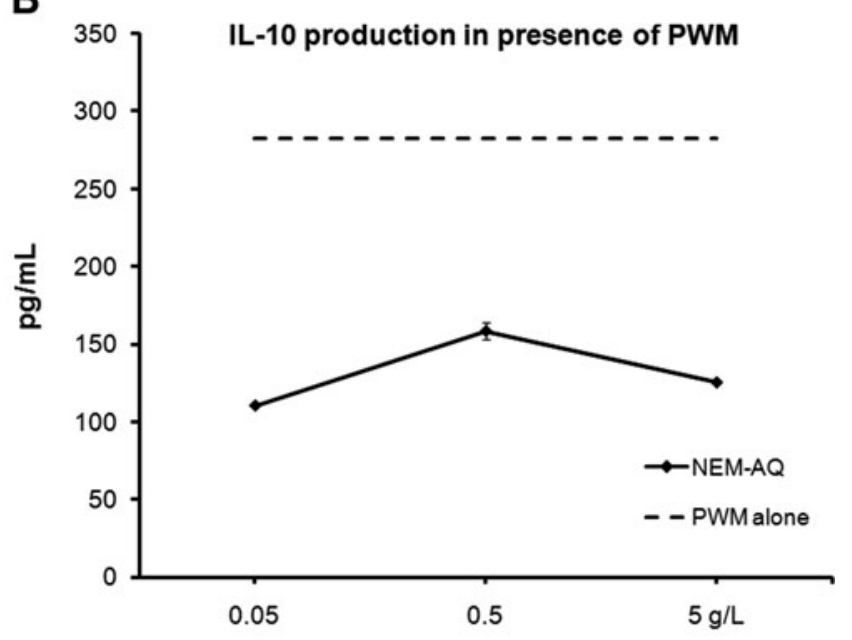

D

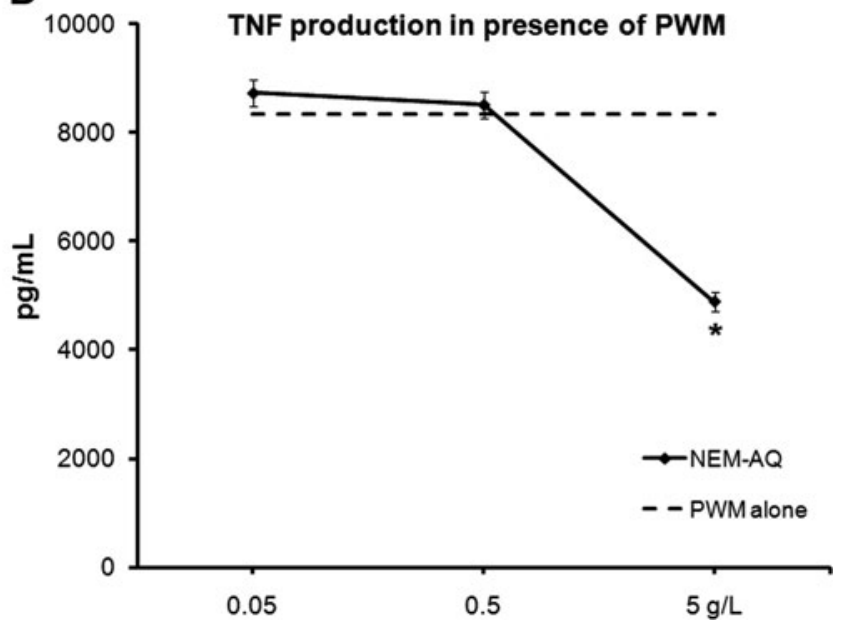

FIG. 3. Effects of NEM-AQ on production of the cytokines (A) IL-6, (B) IL-10, (C) IFN- $\gamma$, and (D) TNF- $\alpha$ by 4-day peripheral blood mononuclear cell cultures simultaneously exposed to PWM. Supernatants from 4-day peripheral blood mononuclear cell cultures were simultaneously assayed for the presence of cytokines using a flow cytometry-based assay. (A) IL-6 production in cultures exposed to serial dilutions of NEM-AQ showed a strong increase at the highest dose tested $(5 \mathrm{~g} / \mathrm{L})$, whereas lower concentrations of NEM-AQ had no effect compared with IL6 production in cultures exposed to PWM alone. (B) All three concentrations of NEM-AQ decreased IL-10 production in the presence of PWM about twofold. (C) Production of IFN- $\gamma$ by 4-day cultures exposed to serial dilutions of NEM-AQ in the presence of PWM was affected differently depending on the concentration of NEM-AQ. These changes were not statistically significant. (D) In the presence of PWM, TNF- $\alpha$ production by 4-day cultures was decreased by the highest concentration of NEM-AQ, whereas lower concentrations of NEM-AQ had no effect on TNF- $\alpha$ production. Statistically significant differences are indicated $(* P<.05)$. The results shown are mean \pm SD values from a representative of three separate lymphocyte proliferation cultures using cells from three different donors.

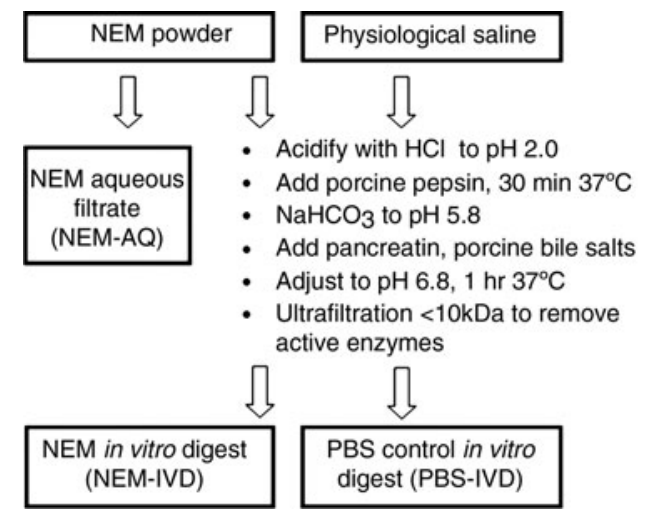

FIG. 4. Diagram outlining the in vitro digestion procedure. Based on methods published in the literature, ${ }^{11-13}$ a stepwise process was performed that incorporated digestive enzymes derived from pig (porcine) and $\mathrm{pH}$ adjustments in order to mimic the digestive processes occurring in the stomach and small intestine. The final digested product was returned to physiological $\mathrm{pH}$ and subjected to size-exclusion centrifugation using a $10-\mathrm{kDa}$ filtration column in order to remove the porcine enzymes. This process was performed with NEM-AQ, resulting in the product referred to as in vitro-digested NEM (NEM-IVD), as well as with phosphate-buffered saline (PBS), resulting in the product referred to as PBS-IVD. 


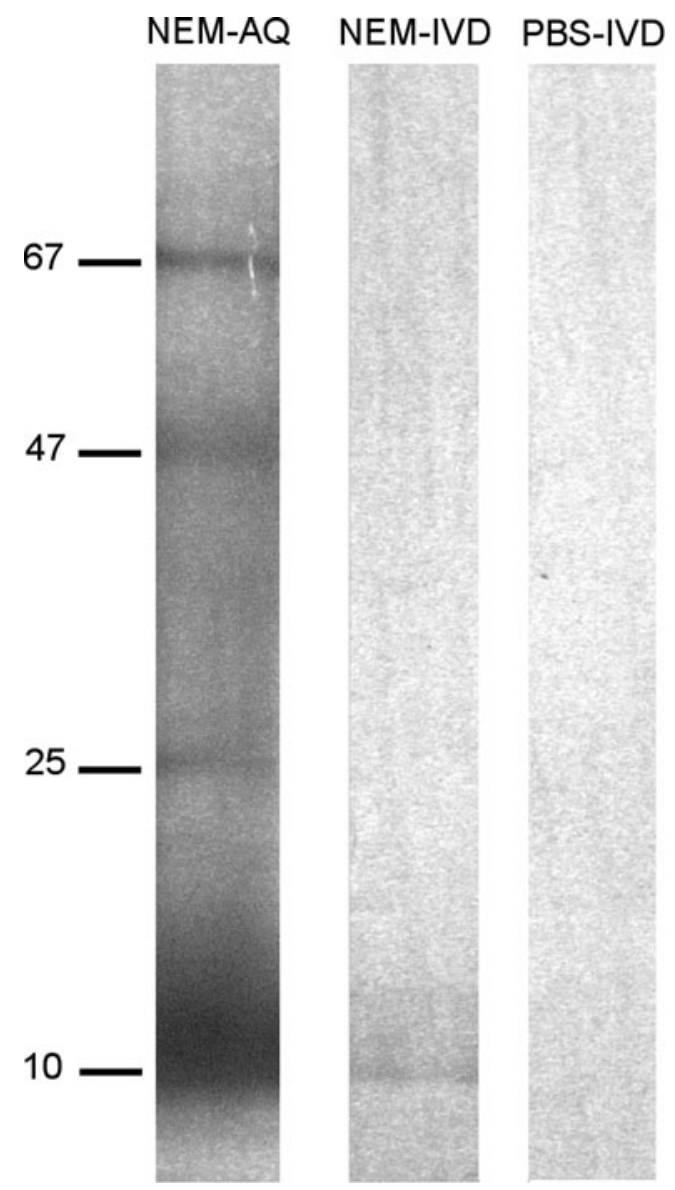

FIG. 5. Sodium dodecyl sulfate-polyacrylamide gel electrophoresis separation of NEM-AQ, NEM-IVD and PBS-IVD. Samples were separated on a $4-15 \%$ polyacrylamide gel using denaturing conditions, and bands were visualized by silver stain. Molecular sizes of major bands in the NEM-AQ lane are indicated in $\mathrm{kDa}$ on the left-hand side. This presentation of the data is used to show the effects of the in vitro digestion of NEM on the molecular weights of the resulting digested protein products. This comparison shows a loss of higher-molecular-weight products when comparing NEMIVD with the undigested NEM-AQ. Within the sensitivity of sodium dodecyl sulfate-polyacrylamide gel electrophoresis and silver stain methods, no residual enzymes or breakdown products from the in vitro digestion process were detectable in the PBS-IVD sample.

showed a reduction of high-molecular-weight material in the NEM-IVD sample (Fig. 5) compared with the NEM-AQ sample, whereas the PBS-IVD sample did not show any detectable protein.

\section{Suppression of TNF- $\alpha$ production resulting from treatment of PBMCs with NEM-IVD}

In the context of the mitogen PWM, reflecting an in vitro model of the cellular collaborations in lymphoid tissue, the native NEM-AQ showed an anti-inflammatory effect only at the highest dose used, and the effect returned to baseline at the lower doses. In contrast, NEM-IVD showed significant anti-inflammatory properties across a wide dose range with

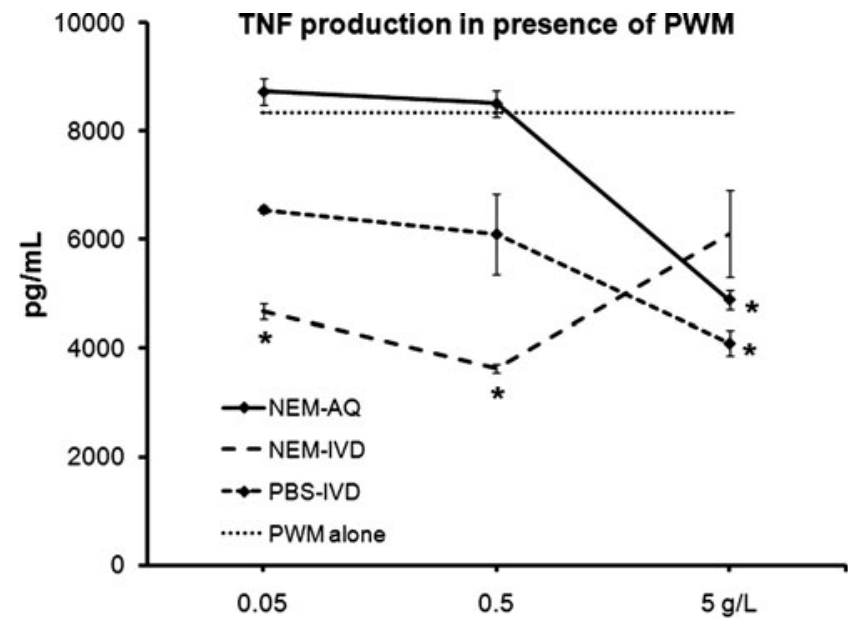

FIG. 6. Comparison of the effect of NEM-AQ, NEM-IVD, and PBS-IVD on production of the cytokine TNF- $\alpha$ by 4-day peripheral blood mononuclear cell cultures simultaneously exposed to PWM. TNF- $\alpha$ production by cultures exposed to PWM was most affected by the NEM-IVD extract. At the highest dose of extracts tested $(5 \mathrm{~g} / \mathrm{L})$, all three products produced a similar reduction in TNF- $\alpha$ production of $30-40 \%$. However, it can be argued that this high dose is beyond a physiological relevant dose after consumption. Therefore, the data for the two lower doses may be more relevant for predicting in vivo outcomes. At the two lowest doses of extracts tested, a different picture emerged. Here NEM-AQ did not affect TNF- $\alpha$ production, showing cytokine levels similar to baseline (cultures exposed to PWM alone). The PBS-IVD extract showed some activity, lowering TNF- $\alpha$ production. However, NEM-IVD had the greatest effect in lowering TNF- $\alpha$ production. At the 0.5 and $0.05 \mathrm{~g} / \mathrm{L}$ concentrations, NEM-IVD lowered TNF- $\alpha$ production by $55 \%$ and $45 \%$, respectively. Statistically significant differences are indicated $(* P<.05)$. The results shown are mean \pm SD values from a representative of three separate lymphocyte proliferation cultures using cells from three different donors.

respect to TNF- $\alpha$ production (Fig. 6). The in vitro-digested saline control (PBS-IVD) had some effects on PBMC culture proliferation and cytokine production that were different from those of saline alone, suggesting the presence of residual material derived from the in vitro digestion process. The effects seen at the two lower doses may be most relevant for predicting in vivo outcomes. The data showed that in the presence of PWM, NEM-AQ had only a minor effect on TNF- $\alpha$ production, whereas NEM-IVD showed $45-55 \%$ suppression of TNF- $\alpha$ production $(P<.04)$.

\section{DISCUSSION}

Osteoarthritis is often considered a local problem centered on the specific target area where bone and joint degradation is seen, such as, for example, a knee. However, osteoarthritis is a systemic disease, involving immune dysregulation and altered cytokine profile (Fig. 7). In particular, $\mathrm{T}$ cells likely play an important role in the pathogenesis and progression of osteoarthritis. Osteoarthritis involves infiltrating monocytes producing TNF- $\alpha .{ }^{15}$ It is also believed that peripheral blood leukocytes, which travel through the tissues of inflamed joints, are activated 


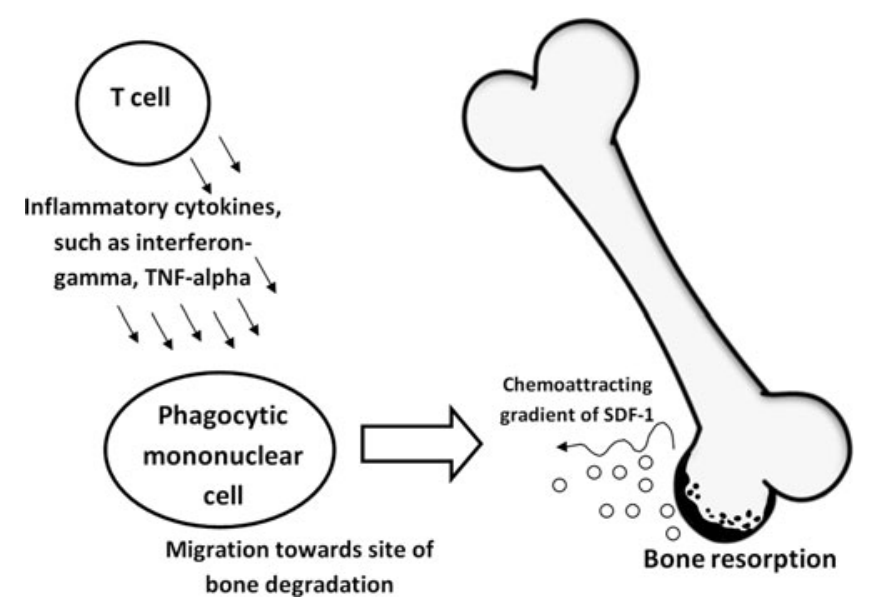

FIG. 7. Diagram showing arthritic mechanisms of action involving T-cell-derived inflammatory cytokines in activation and migration of phagocytic mononuclear cells into areas of bone destruction. SDF-1, stromal cell-derived factor- 1 .

through exposure to locally produced mediators of inflammation (i.e., IL-1 $\beta$, TNF- $\alpha$, etc.). ${ }^{16} \mathrm{We}$ were therefore interested in studying cytokine production in peripheral blood mixed cultures including monocytes. This was accomplished through an extended proliferation/cytokine assay, where both digested and undigested NEM preparations with appropriate controls were tested in serial dilutions in the presence and absence of mitogens. Two mitogens were tested in parallel: PHA, which is a T-cell mitogen that will induce T-cell proliferation, and PWM, which is a mitogen that requires the collaboration of $\mathrm{T}$ cells, B cells, and monocytes in the culture.

The significance of the data must be interpreted in light of the specificity of the culture conditions in the presence of the two separate mitogens, PHA and PWM, as well as the importance of the sequence in which stimulating agents were added. PWM is an aqueous extract from Phytolacca americana (pokeweed) that has mitogenic properties that involve mechanisms closely mimicking events in lymph nodes and other immune tissue where antigen presentation leads to co-stimulation and collaboration of multiple cell subsets. The mechanisms involve leukocyte aggregation. ${ }^{17}$ RNA synthesis precedes DNA synthesis by 24 hours, after which cell division begins, involving up to $60 \%$ of the peripheral blood lymphocyte fraction. ${ }^{18}$ The activation process involves $\mathrm{T}$ lymphocytes, $\mathrm{B}$ lymphocytes, and phagocytic mononuclear cells ${ }^{19}$ in tandem and generates both $\mathrm{T}$ cell- and $\mathrm{B}$ cell-derived cytokines, ${ }^{20}$ leading to generation of immunoglobulin-secreting plasma cells ${ }^{21}$ and a shift in CD45 isoform expression indicative of plasma cell differentiation. ${ }^{22}$ In contrast, an extract from Phaseolus vulgaris (red kidney bean) called PHA predominantly activates $\mathrm{T}$ lymphocytes, even though some Bcell activation can be seen as a result of the activated $T$ cells triggering some B lymphocytes into proliferation. ${ }^{23}$ Therefore, these two mitogens were used as a method to shed light on events that are strictly $\mathrm{T}$ cell mediated versus events that require complex cellular collaboration (B cells and $\mathrm{T}$ cells).

The reduction of proliferation in NEM-treated cultures should not be seen as a suppression of a mitogenic response, but rather as evidence that NEM has leukocyte signaling properties of its own. The sequential addition of NEM first, followed by mitogens after 5 minutes, allowed compounds in NEM to engage signaling in target cells so when the mitogens were subsequently added the resultant signal was diminished.

Because NEM affected both PHA and PWM mitogenicity, but in different ways, this finding suggests specific mechanisms, including that NEM contains compounds directly able to modulate T-cell activation, and that NEM also has immune-modulating properties in the context of a more physiological activation process, such as in the PWM model of lymphocyte activation.

Cytokine production was affected in the cultures, with significant changes in three inflammatory cytokines: IL-6, IFN- $\gamma$, and TNF- $\alpha$. It is interesting that this did not change when NEM was passed through the in vitro digestion protocol, except for TNF- $\alpha$. The reduction in TNF- $\alpha$ production with NEM-IVD was seen at 100-fold lower doses than with undigested NEM. In the case of PWM stimulation, NEMIVD showed anti-inflammatory properties by drastically reducing the production of TNF- $\alpha$, in contrast to the mild increase in TNF- $\alpha$ production when cells were pretreated with NEM-AQ. Thus, the in vitro digestion potentiated the anti-inflammatory action of NEM, so that much lower doses of NEM-IVD were seen to produce similar effects as 25 -fold higher doses of NEM-AQ. This is relevant for suggesting anti-inflammatory mechanisms in vivo after consumption of NEM and subsequent digestion in the stomach.

The dose-responses seen in the different assays were in several cases nonlinear. This may be attributed to several confounding factors associated with the highest dose, suggesting that the biological effects observed at lower doses should receive the most attention. It may be argued that the highest dose we used $(5 \mathrm{~g} / \mathrm{L})$ exceeds a likely physiologically relevant dose. However, it may also be argued that this dose may be reached locally along the intestinal mucosa after consumption. We suggest that the highest dose $(5 \mathrm{~g} / \mathrm{L})$ is quite high and that the biological responses seen at the two lower doses may be more relevant for predicting in vivo outcomes. There is also the possibility that calcium may have been an issue at the highest dose. NEM contains some calcium from unseparated eggshell, so for the most part this will be insoluble calcium carbonate. At the $5 \mathrm{~g} / \mathrm{L}$ dose of NEM, the amount of calcium present may still be sufficient to interfere with cellular signaling. Further dilution of NEM may dilute calcium to insignificant levels. As it is therefore unlikely that calcium contributed to the different responses seen at the lowest dose, it cannot be completely ruled out as a potential mechanism of disturbing or abrogating cellular signaling at the highest dose used.

In the case of the TNF- $\alpha$ cytokine testing, the most interesting difference between the effects of NEM-AQ and NEM-IVD was the reduction in TNF- $\alpha$ production in the 
presence of PWM that occurred with all three concentrations of NEM-IVD. These reductions in TNF- $\alpha$ production were strongest with the lowest doses of NEM-IVD, and this effect was opposite to that seen for NEM-AQ. Although the in vitro digestion procedure introduced compounds that were not completely removed by the size exclusion centrifugation step and that possessed bioactivity, the digestion process nevertheless increased the ability of NEM-AQ to reduce TNF- $\alpha$ production in 4-day PBMC cultures in the presence of PWM. The effect of NEM-IVD on TNF- $\alpha$ production in the presence of PWM was also different from the effect resulting from treatment of PBMC cultures with PBS-IVD and points to activities unique to the NEM-IVD product.

In particular, the result of NEM-AQ and NEM-IVD reducing TNF- $\alpha$ production is of interest in terms of identifying mechanisms of action pertaining to arthritis conditions because this cytokine is known to attract cell infiltration into arthritic joints and contribute to the inflammation within the joint.

Comparison of the effects of NEM-AQ and NEM-IVD on cytokine production by PBMC cultures revealed some differences that could not be entirely attributed to activities derived from the in vitro digestion process itself (such as enzyme breakdown products or residual bile salts that were not removed by the size exclusion centrifugation step). This unique effect of NEM-IVD with respect to TNF- $\alpha$ clearly warrants efforts to further investigate the effects of in vitro digestion on NEM. This is of particular importance as several biological TNF- $\alpha$-inhibiting drugs have proven quite effective in treating arthritis but have been shown to have infrequent but often severe side effects. ${ }^{24} \mathrm{~A}$ treatment, such as NEM, that has immunomodulatory properties that are likely more diffuse could potentially avoid the unfortunate side effects of the currently available biological drugs.

\section{ACKNOWLEDGMENTS}

This study was performed at NIS Labs, an independent research lab specializing in natural products research, and was sponsored by ESM Technologies, LLC.

\section{AUTHOR DISCLOSURE STATEMENT}

K.F.B. and G.S.J. are employed by NIS Labs, an independent contract research laboratory. K.J.R. is employed by ESM Technologies, LLC in the function of Director of Scientific and Regulatory Affairs. The authors have no other financial interest in the subject matter.

\section{REFERENCES}

1. Gonzalez EA: The role of cytokines in skeletal remodeling: possible consequences for renal osteodystrophy. Nephrol Dial Transplant 2000;15:945-950.

2. Komano Y, Nanki T, Hayashida K, Taniguchi K, Miyasaka N: Identification of a human peripheral blood monocyte subset that differentiates into osteoclasts. Arthritis Res Ther 2006; 8:R152.
3. Tsutsumi H, Tanaka T, Ohashi N, Masuno H, Tamamura H, Hiramatsu K, Araki T, Ueda S, Oishi S, Fujii N: Therapeutic potential of the chemokine receptor CXCR4 antagonists as multifunctional agents. Biopolymers 2007;88:279-289.

4. Santiago B, Baleux F, Palao G, Gutierrez-Canas I, Ramirez JC, Arenzana-Seisdedos F, Pablos JL: CXCL12 [SDF-1] is displayed by rheumatoid endothelial cells through its basic amino-terminal motif on heparan sulfate proteoglycans. Arthritis Res Ther 2006;8:R43.

5. Ruff KJ, Devore DP, Leu MD, Robinson MA: Eggshell membrane: a possible new natural therapeutic for joint and connective tissue disorders. Results from two open-label human clinical studies. Clin Interv Aging 2009;4:235-240.

6. Ruff KJ, Winkler A, Jackson RW, DeVore DP, Ritz BW: Eggshell membrane in the treatment of pain and stiffness from osteoarthritis of the knee: a randomized, multicenter, double-blind, placebo-controlled clinical study. Clin Rheumatol 2009;28:907914.

7. Wong M, Hendrix MJC, von der Mark K, Little C, Stern R: Collagen in the egg shell membranes of the hen. Dev Biol 1984;104:28-36.

8. Baker JR, Balch DA: A study of the organic material of hen'segg shell. Biochem J 1962;82:352-361.

9. Long FD, Adams RG, DeVore DP, inventors; New Life Resources, LLC, assignee: Preparation of hyaluronic acid from eggshell membrane. U.S. Patent 6,946,551. September 20, 2005.

10. Picard J, Paul-Gardais A, Vedel M: Sulfated glycoproteins from egg shell membranes and hen oviduct. Isolation and characterization of sulfated glycopeptides. Biochim Biophys Acta 1973;320: 427-441.

11. Yun S, Habicht J-P, Miller DD, Glahn RP: An in vitro digestion/ Caco-2 cell culture system accurately predicts the effects of ascorbic acid and polyphenolic compounds on iron bioavailability in humans. J Nutr 2004;134:2717-2721.

12. Boyer J, Brown D, Liu RH: In vitro digestion and lactase treatment influence uptake of quercitin and quercetin glucoside by the Caco-2 cell monolayer. Nutr J 2005;4:1-15.

13. Toor RK, Savage GP, Lister CE: Release of antioxidant components from tomatoes determined by an in vitro digestion method. Int J Food Sci Nutr 2009;60:119-129.

14. Jensen GS, Benson KF, Carter SG, Endres JR: GanedenBC30 cell wall and metabolites: anti-inflammatory and immune modulation effects in vitro. BMC Immunol 2010;11:15.

15. Sakkas L, Platsoucas C: The role of T cells in the pathogenesis of osteoarthritis. Arthritis Rheum 2007;56:409-424.

16. Attur M, Belitskaya-Lévy I, Oh C, Krasnokutsky S, Greenberg J, Samuels J, Smiles S, Lee S, Patel J, Al-Mussawir H, McDaniel G, Kraus VB, Abramson SB: Increased interleukin- $1 \beta$ gene expression in peripheral blood leukocytes is associated with increased pain and predicts risk for progression of symptomatic knee osteoarthritis. Arthritis Rheum 2011;63:1908-1917.

17. Börjeson J, Reisfeld R, Chessin LN, Welsh PD, Douglas SD: Studies on human peripheral blood lymphocytes in vitro. I. Biological and physicochemical properties of the pokeweed mitogen. J Exp Med 1966;124:859-872.

18. Chessin LN, Börjeson J, Welsh PD, Douglas SD, Cooper HL: Studies on human peripheral blood lymphocytes in vitro. II. Morphological and biochemical studies on the transformation of lymphocytes by pokeweed mitogen. J Exp Med 1966;124:873884. 
19. de Vries E, Lafeber GJ, van der Weij JP, van Buijsen AC, Leijh PC, Cats A: Pokeweed-mitogen induced lymphocyte proliferation: the effect of stimulation on mononuclear phagocytic cells. Immunology 1980;40:177-182.

20. Weksler ME, Kuntz MM: Synergy between human $\mathrm{T}$ and $\mathrm{B}$ lymphocytes in their response to phytohaemagglutinin and pokeweed mitogen. Immunology 1976;31:273-281.

21. Hammarström L, Bird AG, Britton S, Smith CI: Pokeweed mitogen induced differentiation of human B cells: evaluation by a protein A haemolytic plaque assay. Immunology 1979;38:181-189.
22. Jensen GS, Poppema S, Mant MJ, Pilarski LM: Transition in CD45 isoform expression during differentiation of normal and abnormal B cells. Int Immunol 1989;1:229-236.

23. Greaves M, Janossy G, Doenhoff M: Selective triggering of human $\mathrm{T}$ and $\mathrm{B}$ lymphocytes in vitro by polyclonal mitogens. J Exp Med 1974;140:1-18.

24. Hochberg MC, Lebwohl MG, Plevy SE, Hobbs KF, Yocum DE: The benefit/risk profile of TNF-blocking agents: findings of a consensus panel. Semin Arthritis Rheum 2005;34: 819-836. 